\title{
Convencionalidade na Aquisição de Verbos: Estudo Comparativo das Análises Dicotômicas e Contínuas
}

\author{
Jaqueline de Carvalho Rodrigues ${ }^{1}$ \\ Lauren Tonietto \\ Tania Mara Sperb \\ Maria Alice de Mattos Pimenta Parente \\ Universidade Federal do Rio Grande do Sul
}

\begin{abstract}
RESUMO - A convencionalidade é um princípio pragmático da aquisição lexical que possibilita a adequação das palavras ao contexto. O objetivo deste estudo foi comparar dois tipos de análise - dicotômica e contínua - da convencionalidade dos verbos, obtidos por meio de uma tarefa de nomeação de ações composta por 17 vídeos breves, aplicada a 37 crianças com idades entre 2:0 e 3:0 e 43 crianças entre 3:1 e 4:5. Foram comparadas duas análises: (1) dicotômica-verbos classificados como não convencionais ou convencionais; e (2) contínua - escores entre 1 e 5, obtidos por escala likert. Os resultados mostraram que as duas formas de análise diferenciam os grupos etários, embora a análise contínua apresente vantagens, revelando mais detalhes do desenvolvimento do léxico de verbos.
\end{abstract}

Palavras-chave: convencionalidade; aquisição lexical; verbos; crianças; escala Likert.

\section{Conventionality in Verb Acquisition: A Comparative Study of Dichotomic and Continuous Analysis}

\begin{abstract}
Conventionality is a pragmatic principle of lexical acquisition which makes the adequacy of words to the context possible. The aim of this study was to compare dichotomic and continuous analysis in the classification of this pragmatic principle. The data were obtained by an actions naming task composed of 17 brief videos, applied to 37 children aged between 2:0 and 3:0 and 43 children aged between 3:1 and 4:5. Two analyses were compared: (1) dichotomic - verbs classified as non conventional or conventional; and (2) continuous, scores between 1 and 5, obtained using a Likert scale. The results showed that the two kinds of analysis distinguish the age groups, although the continuous analysis presents advantages, revealing more details about the verbs lexical development.
\end{abstract}

Keywords: conventionality; lexical acquisition; verbs; children; Likert scale.

No contexto da aquisição lexical, vários são os métodos de investigação do desenvolvimento da linguagem nas crianças, como check lists, registros de falas espontâneas ou dirigidas a priori, situações de narrativas, com ou sem imagens, e tarefas de nomeação. É bastante conhecido o fato que a diversidade de situações das tarefas pode produzir diferentes resultados (por exemplo, Tardif, Gelman \& Xu, 1999). Entretanto, o viés produzido pela forma de classificação das respostas infantis tem sido menos explorado.

Nas tarefas de nomeação de figuras e de ações, frequentemente o critério utilizado para classificar as respostas das crianças é a análise dicotômica do tipo certo ou errado. Por si só, este critério apresenta dificuldades, pois uma mesma representação figurativa pode ser nomeada de diferentes formas, por exemplo, um animal de quatro patas pode ser nomeado como cão, animal, pastor alemão ou Totó, sem deixar de ser uma forma adequada de nomeação no contexto em que foi dita.

Estudos de Clark (1988) e Duvignau (2003) sugerem que, no período de aquisição do léxico, a forma de avaliação das

1 Endereço para correspondência: UFRGS-Instituto de Psicologia, Rua Ramiro Barcelos, $n^{\circ} 2600$, sala 114, Bairro Santa Cecília. Porto Alegre, RS. CEP 90035-003. E-mail: jaquecarvalhorodrigues@gmail.com respostas infantis deve considerar uma estratégia frequente, denominada "aproximação semântica", definida como uma forma alternativa utilizada pela criança para se expressar, substituindo palavras desconhecidas por outras de significados semelhantes, por vezes ampliando o significado de determinadas palavras. Por exemplo, para a ação de estourar um balão, a expressão "quebrou o balão" foi emitida com certa frequência por crianças com idades entre dois e quatro anos nos estudos de Tonietto (2005) e Tonietto, Parente, Duvignau, Gaume e Bosa (2007). Tais "aproximações semânticas" da linguagem infantil mostram a flexibilidade linguística das crianças e sua possibilidade em utilizar itens já disponíveis em seu léxico, ainda em fase de construção. Posteriormente, com maior experiência linguística, as formas alternativas ou menos convencionais dão lugar a formas mais convencionais, utilizadas pelos falantes adultos, como "estourou o balão". Assim, a aquisição da convencionalidade é um fator importante para o desenvolvimento linguístico.

Embora "quebrar o balão" não seja a forma considerada convencional de acordo com o padrão adulto para nomear a ação de "estourar um balão", esse léxico aproximativo é compreendido pelos familiares e interlocutores. De certa maneira, o uso de aproximações semânticas (também frequente em um aprendiz de uma nova língua) pode causar 
certa estranheza para o ouvinte adulto, mas não impede a comunicação infantil. Entretanto, um dos princípios de uma comunicação efetiva é o uso das palavras convencionais para objetos e fatos, definidas conforme as regras de cada cultura.

$\mathrm{Na}$ avaliação da convencionalidade, a classificação dicotômica do tipo certo/errado, além de teoricamente inadequada, pode apresentar baixa concordância entre juízes. O presente estudo discute a forma de classificação da convencionalidade de verbos em uma tarefa de nomeação e compara dois tipos de análises desta característica - uma dicotômica e outra contínua.

\section{Desenvolvimento da Convencionalidade}

A convencionalidade é um princípio pragmático proposto inicialmente por Clark (1988, 1997), que define o uso convencional das palavras em uma determinada comunidade linguística. Glock (2003) destacou que as regras da linguagem devem ser compartilhadas entre os falantes. Para que uma comunicação seja efetiva, é importante que os falantes escolham e usem as mesmas palavras para um determinado significado (Diesendruck, 2005).

A convencionalidade é um dos princípios da aquisição lexical descritos por Golinkoff, Mervis e Hirsh-Pasek (1994), também observada na aquisição de verbos (Golinkoff, Hirsh-Pasek, Mervis, Frawley \& Parillo, 1995). Para Tomasello (2003), uma das tarefas essenciais no aprendizado da linguagem é justamente a adequação do uso das palavras ao contexto, ou seja, a criança precisa aprender que existem formas convencionais para referir-se a determinadas situações. Cedo no desenvolvimento, a criança espera que seu interlocutor fale de acordo com o que é convencional e percebe as intenções dos falantes quando nomeiam objetos (Akhtar, Carpenter \& Tomasello, 1996). Além disso, as crianças discriminam entre formas conhecidas e desconhecidas do seu interlocutor (Diesendruck \& Markson, 2001) e, em torno de quatro anos, já fazem hipóteses sobre o uso convencional dos falantes (Diesendruck, 2005).

Durante o desenvolvimento, em vários aspectos da linguagem a produção é mais tardia do que a compreensão, ocorrendo o mesmo com a convencionalidade. Até os quatro anos, a criança ainda está adquirindo o uso convencional das palavras, diminuindo a produção de aproximações semânticas com a idade (Tonietto et al., 2007). Em um estudo transcultural comparando a nomeação de verbos de crianças falantes do português com as falantes de mandarim, observou-se que o aprendizado da convencionalidade parece ter um curso semelhante nas duas culturas bastante contrastantes (Ping, Tonietto, Parente, Duvignau \& Gaume, 2009). Contudo, o léxico mais diverso e extenso das crianças chinesas pode ser explicado pelo grau de concretude dos verbos em mandarim (Ma et al., 2006; Tardif, 2006).

\section{A aquisição dos verbos}

A polêmica afirmação de Gentner (1978) sobre a existência de um "viés do substantivo" (noun bias) - segundo a qual os substantivos são aprendidos com maior facilidade do que os verbos - despertou um grande interesse pelo estudo da aquisição de verbos nas crianças. Diversos aspectos nesta aquisição foram levantados, entre outros: o desenvolvimento morfológico (Wagner, Swensenb \& Naigles, 2009) e a influência da facilitação (Keren-Portnoy, 2006; Kidd, Lieven \& Tomasello, 2006).

Para Gentner (1978), as crianças aprendem, primeiramente, o significado dos substantivos porque eles designam objetos, itens mais concretos e mais facilmente percebidos do que os eventos que descrevem ações. Além disso, os verbos são termos relacionais, referem-se a conceitos mais abstratos e menos coesos. Consequentemente, os limites que diferenciam um verbo de outro são menos claros e de mais difícil aprendizado.

Gentner e Rattermann (1991) analisaram a aquisição em várias línguas e propuseram que o viés do substantivo seria universal, fato confirmado por outros estudos no inglês (Fenson et al., 1994) e no italiano (Caselli et al., 1995). Entretanto, estudos transculturais questionaram a hipótese do "viés do substantivo", mostrando que em algumas línguas os verbos estão presentes desde muito cedo e em grande quantidade, como observado no coreano (Gopnik, Choi \& Baumberger, 1996), no chinês (Ma et al. 2006; Tardif, 1996; Tardif, Gelman \& Xu, 1999) e no japonês (Fernald \& Morikawa, 1993). No português brasileiro, dois estudos mostram resultados diferentes. Vidor (2008) encontrou um número maior de substantivos enquanto Befi-Lopes, Cáceres e Araújo (2007) evidenciaram uma prevalência de verbos em sua amostra. Essas diferenças podem estar relacionadas às idades das crianças pesquisadas, já que a amostra de Vidor (2008) inclui crianças entre um e três anos de idade, enquanto Befi-Lopes et al. (2007) pesquisaram crianças entre dois e cinco anos.

Estudos neuropsicolinguísticos têm proposto uma dissociação entre verbos e substantivos, ao evidenciar diferentes processamentos cerebrais requeridos para cada classe gramatical em adultos (Cappa \& Perani, 2002; Damasio \& Tranel, 1993). Estudos experimentais também têm confirmado uma dificuldade na aquisição de verbos. Até os 30 meses, as crianças encontram muito mais dificuldade em mapear verbos a ações do que substantivos a objetos, apesar de bem precocemente, já aos 10 meses, a criança conseguir identificar qual palavra determina a ação (Maguire, Hirsh-Pasek \& Golinkoff, 2006). Isso quer dizer que no final do primeiro ano de vida as crianças já compreendem que algumas palavras designam ações, entretanto vários anos serão necessários para utilizar adequadamente a palavra apropriada para uma ação específica.

Diferentemente de uma dissociação, Maguire et al. (2006) propõem que o aprendizado, tanto de substantivos como de verbos, depende de diferentes parâmetros contínuos. Nessa perspectiva, os verbos encontram-se no pólo mais complexo. Os parâmetros desenvolvimentais propostos pelos autores são agrupados na sigla SICI (Shape, Individuation, Concreteness e Imageability). O primeiro parâmetro, "forma" (shape) refere-se ao fato que, primeiramente, a criança adquire seu léxico por meio de sua experiência perceptual. A individuação (individuation) é definida como uma maior individualidade perceptual de um item. A concretude (concreteness) refere-se à possibilidade do item de ser visto, escutado ou sentido. A imageabilidade (imageability) é a característica que define a 
maior ou menor facilidade de ser criada uma imagem mental do item (Paivio, Yuille \& Madigan, 1968).

As palavras que denominam formas mais homogêneas são adquiridas anteriormente do que as heterogêneas, que podem ter uma diversidade de formatos. Em geral, os substantivos têm uma forma mais homogênea do que verbos: substantivos concretos, como "xícara", são anteriores aos substantivos abstratos, como "esperança". Da mesma forma, verbos de movimento explícito como "dançar" são anteriores a "pensar" (exemplos dos autores). Os quatro parâmetros que integram o SICI são contínuos que justificam a tardia aquisição de alguns verbos, mas também a aquisição mais tardia de substantivos abstratos.

Esses parâmetros têm se mostrado importantes para explicar a aquisição diferenciada nos estudos translinguísticos. Ma et al. (2006) e Tardif (2006) pediram a nativos anglofônicos e falantes de mandarin para analisarem numa escala Likert o grau de concretude das primeiras palavras adquiridas por crianças dessas duas comunidades linguísticas. Os resultados mostraram um maior grau de concretude nos verbos chineses do que nos ingleses, o que poderia explicar a mais rápida aquisição de verbos pelas crianças chinesas. Na mesma direção, Gillette, Gleitman, Gleitman e Lederer (1999) e Genter e Boroditsky (2001) observaram que crianças apresentam maior facilidade de emparelhar palavras e ações bem concretas.

Os parâmetros incorporados pelo SICI referem-se a aspectos perceptuais do item a ser nomeado e representam uma estratégia inicial no aprendizado das palavras. Primeiramente a criança desenvolve seu léxico de acordo com seu ponto de vista. Apesar de pistas sociais serem básicas para a aquisição da linguagem em geral (Tomasello, 2003), o uso das peculiaridades sociais do significado de palavras são mais tardias. Segundo Hollich et al. (2000), somente por volta dos 19 meses as crianças começam a utilizar pistas sociais para descobrir o significado das palavras. Sendo a ação um evento perceptualmente complexo, a criança precisa inferir a intenção comunicativa e o foco do falante (Tomasello, 2003), assim como a intenção do ator (Poulin-Dubois \& Forbes, 2002). Essas pistas sociais são essenciais para o aprendizado do léxico convencional utilizado por uma determinada comunidade linguística.

\section{A convencionalidade como variável dicotômica e contínua}

Nos estudos de Duvignau (2003), Tonietto (2005), Tonietto et al. (2007) e Ping et al. (2009), a convencionalidade foi tratada como uma variável dicotômica. Assim, os verbos emitidos na Tarefa de Nomeação de Ações (Duvignau, 2004) (ver Instrumentos e Tabela 1) foram classificados como "não convencionais" ou "convencionais". Este tipo de classificação gerou dificuldades teóricas e práticas. Se os demais parâmetros da aquisição lexical, de acordo com o modelo SICI, têm uma característica contínua, é pouco provável que, diferentemente, a convencionalidade seja dicotômica. Também se destaca que, na comunicação cotidiana, algumas emissões (não apenas infantis) geram maior ou menor estranheza, o que conduz a pensar que a classificação de convencionalidade também deve ser representada em um contínuo.
No estudo de Tonietto (2005), várias emissões infantis foram difíceis de classificar como convencionais ou não convencionais, sugerindo a necessidade de estabelecer categorias intermediárias. Por exemplo, para a ação de "estourar o balão" (Tabela 1), o verbo "furar" parece não se enquadrar em uma das categorias: "não convencional" ou "convencional". Assim, visando ao aprimoramento da forma de análise realizada nos estudos prévios sobre convencionalidade dos verbos (Tonietto, 2005; Tonietto et al., 2007), este estudo propõe a discussão de uma forma alternativa de análise da convencionalidade, considerando-a como uma variável contínua, em oposição à dicotomia "não convencional" versus "convencional".

Os objetivos deste estudo são: (1) demonstrar que os dois tipos de análise - dicotômica e contínua - revelam a dinâmica do desenvolvimento da convencionalidade dos verbos; (2) comparar os tipos de análise - dicotômica e contínua - e apontar vantagens e desvantagens; e (3) mostrar que a análise contínua é uma forma mais apropriada de classificação da convencionalidade, uma vez que permite uma análise mais detalhada do desenvolvimento do léxico de verbos.

Esta pesquisa justifica-se pela necessidade de realizar estudos brasileiros sobre o desenvolvimento da convencionalidade, já que não foram localizados estudos sobre esse aspecto na aquisição de verbos. Sabe-se que a dificuldade no uso de termos convencionais em crianças pode refletir uma rigidez cognitiva típica de transtornos do desenvolvimento da linguagem. Assim, faz-se necessário e importante buscar estratégias para a intervenção em crianças que apresentem atrasos ou transtornos da linguagem. Além disso, é necessário buscar um método eficaz de análise da classificação das respostas nos estudos de aquisição lexical para adequá-lo às possibilidades de nomeação da linguagem infantil, que mostram a flexibilidade linguística das crianças, cujo léxico ainda está em construção.

\section{Método}

Este estudo tem um delineamento transversal, quase-experimental, de grupos contrastantes, de acordo com Naschmias e Naschmias (1996).

\section{Participantes}

Participaram desse estudo 80 crianças $(\mathrm{M}=3,09$; $\mathrm{DP}=0,58$ ), divididas em dois grupos etários: (1) 37 crianças com idades entre 2:0 e 3:0 $(M=2,6 ; \mathrm{DP}=0,3)$ e (2) 43 crianças com idades entre $3: 1$ e 4:5 $(\mathrm{M}=3,55 ; \mathrm{DP}=0,31)$. Todas apresentavam desenvolvimento típico e eram frequentadoras de pré-escolas particulares localizadas na Região Metropolitana de Porto Alegre. Não foram incluídas na amostra crianças bilíngues, com diagnóstico de alguma patologia como atraso psicomotor, dificuldades de visão e audição não corrigidas, déficit de atenção, transtornos do desenvolvimento e outras dificuldades cognitivas que pudessem dificultar a comparação com crianças da mesma faixa etária e das mesmas condições socioeconômicas. Para o julgamento das escalas Likert dos verbos participaram 405 estudantes universitários com média 
de idade de 22,56 anos (DP=6,41), de 12 diferentes cursos de graduação e de três diferentes universidades de Porto Alegre.

\section{Instrumentos}

Termo de Consentimento Livre e Esclarecido: A participação neste estudo foi condicionada à assinatura do Termo de Consentimento Livre e Esclarecido pelos pais ou responsáveis pelas crianças e pelos estudantes universitários.

Ficha de Dados Sociodemográficos: Uma Ficha de Dados Sociodemográficos foi preenchida pelos pais das crianças ou responsáveis e pelos estudantes universitários, com o objetivo de caracterizar os participantes e avaliar os critérios de inclusão na amostra.

Tarefa de Nomeação de Ações: O instrumento desenvolvido por Duvignau (2004) é composto por 17 filmetes de ações simples descritas na Tabela 1. Cada filmete tem duração entre 42 segundos a um minuto e 13 segundos, incluindo o tempo de pausa no final de cada ação. Inicialmente, há um breve som indicando o começo da cena. Uma breve música toca, enquanto uma cortina vermelha se abre. Uma mulher utilizando um nariz de palhaço sai detrás da cortina e caminha até uma mesa onde estão dispostos vários objetos. Pega um deles e executa uma ação. Após a apresentação de cada filmete, o participante responde à pergunta: "O que a mulher fez?". Por exemplo, após assistir a um filmete no qual a mulher "descasca uma banana", as crianças respondem: "tirou a roupa da banana, "cortou a banana", "tirou a casca da banana" ou "descascou a banana".

Tabela 1. Filmetes e Ações da Tarefa de Nomeação de Ações (TNRA)

\begin{tabular}{ll}
\hline Filmete & Ação \\
\hline Árvore & Descascar um pedaço de tronco de árvore \\
Balão & Estourar um balão com a mão \\
Banana & Descascar uma banana \\
Cenoura & Descascar uma cenoura \\
Camisa & Descosturar parcialmente a manga de uma camisa \\
Jornal & Rasgar um jornal ao meio \\
Lego & Desmontar um pequeno castelo de lego \\
Laranja & Descascar parcialmente uma laranja \\
Pão1 & Cortar um pão do tipo baguete ao meio com uma \\
Pão2 & serra de pão \\
Pividir um pão do tipo baguete ao meio com as \\
mãos
\end{tabular}

\section{Procedimentos}

Procedimentos Gerais e Éticos: Os participantes deste estudo foram selecionados por meio do procedimento de amostragem não-aleatório, por conveniência (Laville \& Dione, 1999). Participaram apenas as crianças que desejaram assistir aos filmetes e cujos pais autorizaram a participação, formalizada por meio de um Termo de Consentimento Livre e Esclarecido. Esta pesquisa foi aprovada pelo Comitê de Ética em Pesquisa da Universidade Federal do Rio Grande do Sul.

Procedimentos da Tarefa de Nomeação de Ações: A tarefa foi aplicada na própria pré-escola de cada criança, em locais relativamente silenciosos, respeitando as condições de ambiente físico e psicológico, conforme sugerido por Pasquali (2001). Os 17 filmetes foram apresentados individualmente em um computador portátil (notebook) no programa Windows Media Player, em ordem aleatória. Após a apresentação de cada filmete, as crianças responderam à pergunta: "O que a mulher fez?". As respostas foram registradas em protocolo específico da forma mais fidedigna possível, incluindo as frases completas emitidas pelas crianças. Ao demonstrarem cansaço, as crianças foram convidadas a realizar um desenho livre, para o qual foram oferecidas folhas brancas de tamanho A4 e giz de cera.

Procedimentos de categorização das respostas: Os dados registrados nos Protocolos e nas Fichas de Dados Sociodemográficos foram incluídos em uma planilha de dados. Para este estudo foram analisados apenas os verbos. Não foram analisadas respostas que não permitiam reconhecer a ação executada no filmete (por exemplo, "comer" para o filmete Banana, Cenoura, Laranja), respostas sem a categoria gramatical de um verbo (por exemplo, "palhaço", "banana", "pão"), respostas metalinguísticas (por exemplo, "não sei, "é difícil") e respostas gestuais.

Para o critério de convencionalidade foram realizadas duas análises:

Análise dicotômica - Não Convencional versus Convencional: Duas juízas previamente treinadas classificaram cada verbo como Não Convencional (NC) - verbo relevante do ponto de vista semântico, mas não pragmático, ou Convencional $(\mathrm{C})$ - verbo relevante do ponto de vista semântico e pragmático. As divergências foram discutidas até se chegar a um consenso para cada verbo. As crianças receberam escores que variavam de 0 a 17 (um ponto para cada verbo convencional), de acordo com o número de verbos convencionais emitidos.

Análise contínua - Escala Likert de convencionalidade: Todos os verbos emitidos pelas crianças (Tonietto, 2005; Tonietto et al., 2007) na Tarefa de Nomeação de Ações foram listados. Devido à grande extensão da lista de verbos (564 verbos), foram organizadas três escalas Likert de convencionalidade contendo os verbos referentes aos respectivos filmetes: (1) filmetes 1 a 5 (180 verbos); (2) filmetes 6 a 11 (193 verbos); (3) filmetes 12 a 17 (191 verbos). Após a apresentação de cada filmete, estudantes universitários foram convidados a julgar os verbos como: (1) nada convencional; (2) pouco convencional; (3) medianamente convencional; (4) muito convencional; e (5) totalmente convencional; até completar 100 julgamentos para cada verbo. A média dos escores atribuídos pelos estudantes foi calculada, gerando um 
Tabela 2. Medianas, Desvios Interquartílicos, Mínimas e Máximas da Convencionalidade das Crianças na Análise Dicotômica e Contínua

\begin{tabular}{lllllllll}
\hline & \multicolumn{3}{c}{ Análise Dicotômica } & \multicolumn{4}{c}{ Análise Contínua } \\
\hline Grupo Crianças & Md & DI & Min & Max & Md & DI & Min & Max \\
$2: 0-3: 0$ & 8 & 5,5 & 1 & 15 & 3,43 & 0,85 & 2,02 & 4,24 \\
$3: 1-4: 5$ & 12 & 4,25 & 7 & 17 & 3,76 & 0,54 & 2,97 & 4,35 \\
\hline
\end{tabular}

escore de convencionalidade para cada verbo. As médias dos valores de convencionalidade dos verbos foram atribuídas aos verbos emitidos pelas crianças em cada filmete. Uma média total de convencionalidade foi calculdada para cada criança, considerando-se a totalidade dos 17 filmetes, podendo variar, portanto, de 1 a 5 .

\section{Análise dos Dados}

As estatísticas descritivas utilizadas para o detalhamento da amostra foram obtidas com o auxílio de planilhas. Nas análises inferenciais foi utilizado o Pacote Estatístico SPSS (Statistical Package for Social Sciences), Versão 12.0. A correlação das duas formas de análise de convencionalidade - dicotômica e contínua - foi obtida por meio do coeficiente de correlação para postos de Spearmann. A comparação dos grupos de crianças foi realizada por meio do teste não paramétrico $U$ de Wilcoxon-Mann-Whitney (WMW ).

\section{Resultados}

A correlação entre as análises dicotômica e contínua atende ao primeiro objetivo deste estudo: demonstrar que os dois tipos de análise - dicotômica e contínua revelam a dinâmica do desenvolvimento da convencionalidade dos verbos. Conforme pode ser observado na Figura 1, as análises dicotômica e contínua se correlacionaram de forma significativa e positiva $\left(r_{s}=0,634 ; p<0,001\right)$. Este resultado revela que ambas as análises evidenciam a dinâmica do desenvolvimento da convencionalidade dos verbos, que aumenta com a idade.

A comparação das análises do tipo dicotômica e contínua oferece subsídios para os segundo e terceiro objetivos deste estudo: comparar os tipos de análise - dicotômica e contínua - para apontar vantagens e desvantagens; e mostrar que a análise contínua é uma forma mais apropriada de classificação da convencionalidade, uma vez que permite uma análise mais detalhada do desenvolvimento do léxico de verbos.

As medianas, os desvios interquartílicos, as mínimas e máximas do desempenho das crianças quanto à convencionalidade dos verbos nas análises dicotômica e contínua podem ser observados na Tabela 2. As medianas aumentam consideravelmente de um grupo etário (2:0 a 3:0) para o outro ( $3: 1$ a 4:5), passando de 8 para 12 na análise dicotômica e de 3,43 para 3,76 na análise contínua. Estes resultados mostram que a convencionalidade dos verbos aumenta com a idade nos grupos etários estudados.

Observa-se também que, na análise dicotômica, o desvio interquartílico do grupo de crianças de 2:0 a 3:0 foi de 5,5, enquanto a mediana foi de 8 . Este resultado revelou uma gran- de variabilidade dos dados nesta faixa etária quando utilizada a análise da convencionalidade como variável dicotômica, fato que não ocorre com a utilização da análise contínua. No grupo de crianças de 3:1 a 4:5 esta variabilidade não ocorre em nenhuma das formas de análise.

Na comparação das médias de convencionalidade foi encontrada diferença estatisticamente significativa entre os dois grupos de crianças, tanto na análise dicotômica $(U=380,5$; $\mathrm{p}<0,001)$ quanto na análise contínua $(U=489 ; \mathrm{p}=0,003)$. Na Figura 2, observa-se que as crianças de $3: 1$ a 4:5 anos emitiram um maior número de verbos convencionais do que as crianças de 2:0 a 3:0 anos, independente da análise utilizada. A Tabela 3 exemplifica os verbos mais e menos convencionais emitidos pelas crianças na Tarefa de Nomeação de Ações.

\section{Discussão}

O primeiro objetivo deste estudo foi demonstrar que ambas as análises dicotômica (não convencional versus convencional) e contínua (escalas Likert) representam de forma semelhante a dinâmica de desenvolvimento da convencionalidade dos verbos. A correlação estatisticamente significativa e positiva revela que as duas formas de análise demonstram de forma semelhante o curso do desenvolvimento, ou seja, medem de forma semelhante o desenvolvimento da convencionalidade dos verbos nas crianças estudadas.

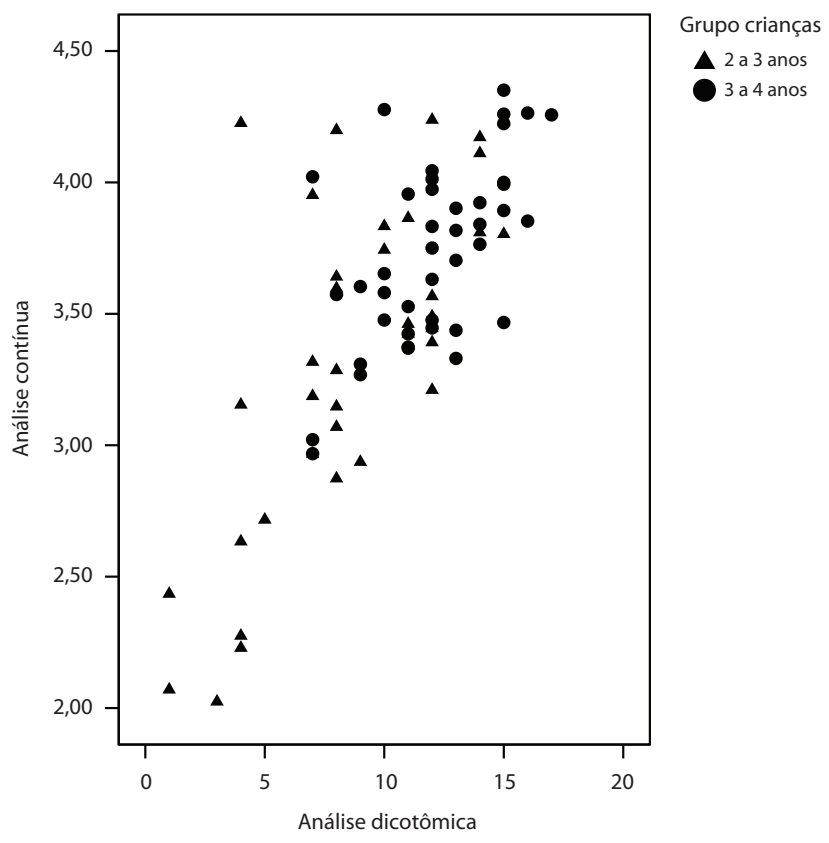

Figura 1. Correlação entre as análises dicotômica e contínua nos grupos de crianças. 

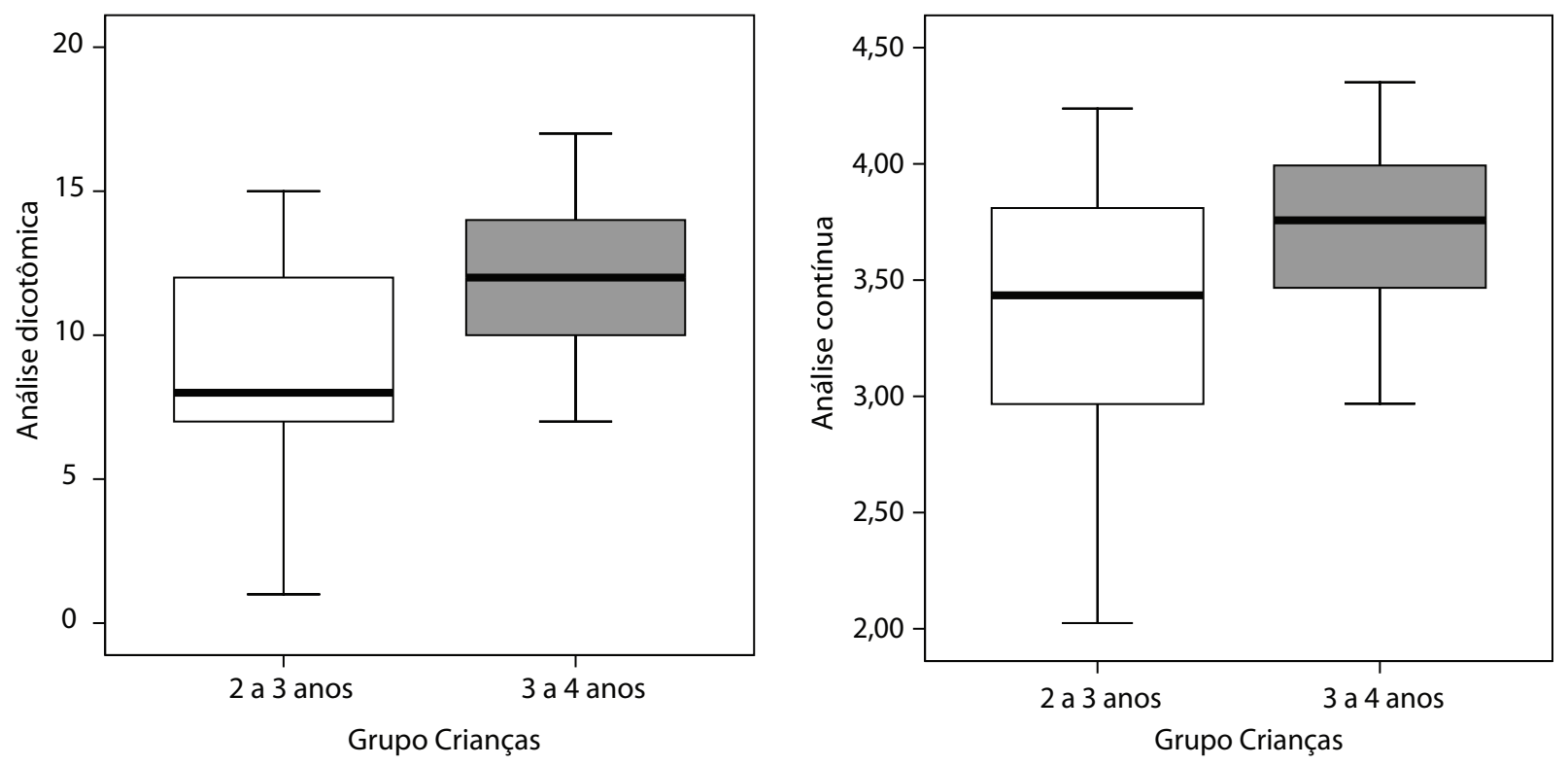

Figura 2. Medianas dos verbos convencionais nas análises dicotômica e contínua nos grupos de crianças de 2 a 3 anos e 3 a 4 anos.

O segundo objetivo foi comparar os dois tipos de análise. A diferença estatisticamente significativa encontrada na convencionalidade dos verbos entre as crianças de 2:0 a 3:0 e 3:1 a 4:5 nos dois tipos de análise evidenciam que ambas as medidas são adequadas para diferenciar os grupos etários. Estes resultados já haviam sido encontrados em Tonietto (2005) e Tonietto et al. (2007). Segundo Golinkoff et al. (1994) e Golinkoff et al. (1995), a convencionalidade é um dos princípios que regem a aquisição lexical relacionada à adequação da linguagem às situações. Da mesma forma, Tomasello (2003) destacou a importância do uso convencional das palavras ao contexto como uma das tarefas essenciais no processo de aprendizagem da linguagem.

O terceiro objetivo do presente estudo foi mostrar que a análise contínua é uma forma mais apropriada de classificação da convencionalidade, uma vez que permite mostrar mais peculiaridades do desenvolvimento do léxico de verbos. Ao comparar o desenvolvimento da convencionalidade utilizando-se dois tipos de análises - dicotômica e contínua - este estudo mostrou inicialmente que, ao utilizar a análise dicotômica, os dados do grupo de crianças de 2:0 a 3:0 apresentam uma assimetria, com um desvio interquartílico muito alto - 5,5 - para a mediana de 8. Dados deste tipo geram certas limitações estatísticas, uma vez que exigem testes não-paramétricos para a comparação das médias. Na análise contínua, o desvio interquartílico dos dois grupos de crianças fica mais harmônico com a mediana, conforme pode ser observado na Tabela 2. Desta forma, os dados podem ser classificados como simétricos e permitem a utilização de testes estatísticos paramétricos, com poderes estatísticos melhores do que os testes não paramétricos. Assim, do ponto de vista estatístico, a análise contínua proposta neste estudo representa uma vantagem sobre a análise dicotômica anteriormente utilizada em Tonietto (2005) e Tonietto et al. (2007). Do ponto de vista do desenvolvimento, reflete o fato de que não existem mudanças repentinas entre uma idade e outra. Ao contrário, o aprendizado da convencionalidade ocorre paulatinamente.

Comparando os desvios interquartílicos de ambas as análises, percebe-se que as crianças de 2:0 a 3:0 tiveram uma maior variabilidade de verbos $(5,5$ na análise dicotômica e 0,85 na análise contínua), enquanto as de 3:1 a 4:5 apresentaram maior homogeneidade em suas respostas (desvio de 4,25 na análise dicotômica e 0,54 na análise contínua). Estes dados revelam que o desenvolvimento da convencionalidade dos verbos apresenta uma grande variabilidade, ou seja, algumas crianças desenvolvem a convencionalidade antes do que outras no curso do desenvolvimento. O estudo de Fenson et al. (1994) discutiu com propriedade a notória variabilidade no desenvolvimento da linguagem. No presente estudo, por exemplo, enquanto algumas crianças na faixa etária de 2 a 3 anos de idade encontram a expressão aproximativa (Duvignau, 2003) ou menos convencional "cortar a banana" ou "abrir a banana" para a ação de "descascar a banana", outras crianças já respondem com "descascar a banana”, expressão considerada convencional (ver Tabela 3 para mais exemplos).

Com a experiência advinda das interações sociais, as crianças ampliam seu vocabulário e percebem quais verbos devem ser utilizados em determinadas ações, isto é, aprendem o que é convencionalmente aceito. Além da vantagem estatística já discutida, a análise contínua permitiu classificar os verbos em cinco categorias, que representam um contínuo de convencionalidade, ao invés das duas categorias anteriormente definidas na análise dicotômica - não convencional versus convencional. Esta maior flexibilidade na classificação dos verbos está de acordo com a noção de parâmetros contínuos que determinam o desenvolvimento linguístico, discutidos amplamente por Maguire et al. (2006). A relação entre um aporte teórico e a forma de mensuração possibilita a elaboração de estudos mais qualitativos que poderão descrever com maior detalhes qual o léxico de verbos de acordo com a faixa etária e seu grau de convencionalidade. 
Tabela 3. Verbos de maior e menor convencionalidade (escala Likert) emitidos pelos dois grupos etários de crianças em cada filmete.

\begin{tabular}{|c|c|c|c|c|}
\hline \multirow{2}{*}{ Ação } & \multicolumn{2}{|c|}{$\begin{array}{c}\text { Grupo etário } \\
2: 0 \text { a } 3: 0 \\
\end{array}$} & \multicolumn{2}{|c|}{$\begin{array}{c}\text { Grupo etário } \\
3: 1 \text { a } 4: 5 \\
\end{array}$} \\
\hline & $+\mathrm{C}$ & $-\mathrm{C}$ & $+\mathrm{C}$ & $-\mathrm{C}$ \\
\hline Descascar tronco & & & & \\
\hline léxico & descascar & picar & descoscar $(12)$ & bagunçar \\
\hline$(\%)$ & (8) & (3) & descascar (12) & (2) \\
\hline Grau de $\mathrm{C}$ & 4,76 & 1,47 & 4,76 & 1,43 \\
\hline \multicolumn{5}{|l|}{ Estourar um balão } \\
\hline léxico & estourar & & estourar & quebrar \\
\hline$(\%)$ & (59) & descascar (3) & (84) & (5) \\
\hline Grau de $\mathrm{C}$ & 4,94 & 1,01 & 4,94 & 1,08 \\
\hline \multirow{2}{*}{\multicolumn{5}{|c|}{$\begin{array}{l}\text { Descascar uma banana } \\
\text { léxico }\end{array}$}} \\
\hline & descascar (22) & quebrar & descascar (35) & quebrar \\
\hline (\%) & & (5) & & (2) \\
\hline Grau de $\mathrm{C}$ & 4,87 & 1,02 & 4,87 & 1,02 \\
\hline \multicolumn{5}{|l|}{ Descascar uma cenoura } \\
\hline léxico & descascar (2) & rasgar & deccoscar (30) & quebrar \\
\hline$(\%)$ & aescascal (22) & (5) & destastal (20) & (5) \\
\hline Grau de C & 4,52 & 1,15 & 4,52 & 1,01 \\
\hline \multicolumn{5}{|l|}{ Descosturar camisa } \\
\hline léxico & rasgar & quebrar & rasgar & quebrar \\
\hline$(\%)$ & (24) & (3) & (44) & (5) \\
\hline Grau de C & 4,34 & 1,07 & 4,34 & 1,07 \\
\hline \multicolumn{5}{|l|}{ Rasgar um jornal } \\
\hline léxico (\%) & $\begin{array}{c}\text { rasgar } \\
(65)\end{array}$ & descascar (3) & rasgar & descascar (2) \\
\hline Grau de C & 4,96 & 1,07 & $\begin{array}{l}(84) \\
4,96\end{array}$ & 1,07 \\
\hline \multicolumn{5}{|l|}{$\begin{array}{l}\text { Desmontar legos } \\
\text { léxico }\end{array}$} \\
\hline léxico & & fazer & & fazer \\
\hline$(\%)$ & desmontar (19) & (3) & desmontar (37) & (5) \\
\hline Grau de C & 4,93 & 1,19 & 4,93 & 1,19 \\
\hline \multirow{2}{*}{\multicolumn{5}{|c|}{$\begin{array}{l}\text { Descascar uma laranja } \\
\text { léxico }\end{array}$}} \\
\hline & descascar (27) & quebrar & descascar (32) & quebrar \\
\hline$(\%)$ & destascal (2i) & (3) & (aesedsedil (32) & (7) \\
\hline Grau de C & 4,92 & 1,14 & 4,92 & 1,14 \\
\hline \multicolumn{5}{|l|}{ Cortar pão com serra } \\
\hline léxico & cortar & descascar (3) & cortar & descascar (5) \\
\hline$(\%)$ & $(51)$ & & $(74)$ & \\
\hline Grau de C & 4,85 & 1,28 & 4,85 & 1,28 \\
\hline \multirow{2}{*}{\multicolumn{5}{|c|}{$\begin{array}{l}\text { Dividir pão com mãos } \\
\text { léxico }\end{array}$}} \\
\hline & tirar pedaco (5) & descascar (5) & partir & descascar (5) \\
\hline$(\%)$ & & & (2) & \\
\hline Grau de C & 2,9 & 1,06 & 4,37 & 1,06 \\
\hline \multicolumn{5}{|l|}{ Esmigalhar um pão } \\
\hline léxico & despedaçar (3) & estourar & despedaçar (5) & descascar (5) \\
\hline $\begin{array}{l}(\%) \\
\text { Grau de } \mathrm{C}\end{array}$ & (4) & $\begin{array}{l}(3) \\
1,13\end{array}$ & 4,04 & 1,41 \\
\hline Amassar folha de papel & & & & \\
\hline léxico & amassar & deccoscar (3) & amassar & estourar \\
\hline$(\%)$ & (46) & descascar (3) & (70) & (2) \\
\hline Grau de $\mathrm{C}$ & 4,81 & 1,10 & 4,81 & 1,27 \\
\hline Picar molho de salsa & & & & \\
\hline léxico & cortar & tirar casca (5) & cortar & tirar \\
\hline$(\%)$ & $(51)$ & tird cased (J) & (79) & (7) \\
\hline Grau de $\mathrm{C}$ & 4,89 & 1,29 & 4,89 & 1,17 \\
\hline Serrar uma tábua & & & & \\
\hline léxico & cortar & & cortar & matar \\
\hline$(\%)$ & (57) & descascar (3) & $(72)$ & (2) \\
\hline Grau de C & 4,13 & 1,14 & 4,13 & 1,06 \\
\hline Despir uma boneca & & & & \\
\hline léxico & tirar rouna (38) & descascar (3) & tirar roun $(77)$ & tirar \\
\hline$(\%)$ & TIrat roupa (30) & aescascar (3) & tirar roupa $(/ / 7)$ & (14) \\
\hline Grau de C & 4,59 & 1,20 & 4,59 & 3,20 \\
\hline Esmagar tomate & & & & \\
\hline Léxico & esmagar & descascar (5) & esmagar & quebrar \\
\hline$(\%)$ & (5) & descascar (5) & (19) & (11) \\
\hline Grau de C & 4,73 & 1,17 & 4,73 & 1,88 \\
\hline Martelar copo & & & & \\
\hline léxico & quebrar & apertar & quebrar & cortar \\
\hline$(\%)$ & (43) & (3) & (74) & (5) \\
\hline Grau de C & 4,43 & 1,37 & 4,43 & 1,51 \\
\hline
\end{tabular}

Nota: $\mathrm{C}=$ convencionalidade $+\mathrm{C}=$ mais convencional; $-\mathrm{C}=$ menos convencional; $\%$ = percentual de crianças que emitiram o léxico indicado. 
No presente estudo, observou-se também que muitos verbos podem ser utilizados para designar ações distintas e podem ser classificados como mais ou menos convencionais dependendo do contexto em que são utilizados. Por exemplo, o verbo "quebrar", emitido em muitos filmetes deste estudo, pode ser classificado como muito convencional na ação de "quebrar um copo", mas como "nada convencional" na ação de "estourar um balão", embora muitas crianças o tenham utilizado para designar esta ação.

Esta flexibilidade para utilizar verbos de diferentes domínios semânticos para expressar uma ação revela que a criança em fase de aquisição da linguagem tem uma flexibilidade linguística e cognitiva. Por outro lado, pode reforçar também a idéia da importância da generalização, já destacada por Vygotsky (1987/1998) e Figueira (1998, 1999). Quando a criança é capaz de utilizar uma mesma palavra em vários contextos significa que ela aprendeu o conceito representado pela palavra e a regra para sua utilização. $O$ posterior aprendizado das exceções às regras e de uma melhor adequação das palavras ao contexto inserirá a criança em sua comunidade linguística.

Este estudo demonstrou que ambas as análises - dicotômica e contínua - da convencionalidade dos verbos permitiram diferenciar grupos de crianças de idades entre 2:0 e 3:0 e entre $3: 1$ e 4:5, revelando diferenças importantes que apontam para um desenvolvimento notável da convencionalidade dentro desta faixa etária. Apesar disso, a análise contínua mostrou-se uma forma aprimorada de análise por suas vantagens estatística, teórica e prática.

A maior flexibilidade na classificação dos verbos em um contínuo de convencionalidade (escala Likert) permitiu uma análise mais criteriosa dos dados, contemplando a visão das teorias sobre conceitos que consideram as categorias como classes abertas (Maguire et al., 2006; Medin \& Schaffer, 1978; Rosch, 1975; Rosch \& Lloyd, 1978). Desta forma, foi possível um melhor enquadramento de verbos anteriormente considerados de difícil classificação.

Para estudos futuros, sugere-se a ampliação da faixa etária das crianças para verificar o curso do desenvolvimento da convencionalidade até o patamar adulto. A comparação de grupos de crianças entre dois e quatro anos com um grupo de adultos já foi publicada em Tonietto (2005) e Tonietto et al. (2007). Além disso, outros estudos translinguísticos poderiam esclarecer semelhanças e diferenças quanto à convencionalidade dos verbos em diferentes culturas.

\section{Referências}

Akhtar, N., Carpenter, M., \& Tomasello, M. (1996). The role of discourse novelty in early word learning. Child Development, 67, 635-645.

Befi-Lopes, D. M., Cáceres, A. M., \& Araújo, K. de. (2007). Aquisição de verbos em pré-escolares falantes do português brasileiro. Revista CEFAC, 9, 444-452.

Cappa, S., \& Perani, D. (2002). The neural correlates of noun and verb processing. Journal of Neurolinguistics, 16, 183-189.

Caselli, M. C., Bates, E., Casadio, P., Fenson, J., Fenson, L., Sanderl, L., \& Weir, J. (1995). A cross-linguistic study of early lexical development. Cognitive Development, 10, 159-199.
Clark, E. V. (1988). On the logic of contrast. Journal of Child Language, 15, 317-335.

Clark, E. V. (1997). Desenvolvimento lexical tardio e formação de palavras. In P. Fletcher \& B. MacWhinney (Eds.), Compêndio da linguagem da criança (M. A. G. Domingues, Trad.) (pp. 323-340). Porto Alegre: Artes Médicas. (Trabalho original publicado em 1995).

Damasio, A. R., \& Tranel, D. (1993). Nouns and verbs are retrieved with differently distributed neural systems. Proceedings of the National Academy of Sciences of the United States of America, 90, 4957-4960.

Diesendruck, G. (2005). The principles of conventionality and contrast in word learning: An empirical examination. Developmental Psychology, 41, 451-463.

Diesenbruck, G., \& Markson, L. (2001). Children's avoidance of lexical overlap: A pragmatical account. Developmental Psychology, 37, 630-641.

Duvignau, K. (2003). Métaphore verbale et approximation. In K. Duvignau, O. Gasquet, \& B. Gaume (Eds.), Regards croisés sur l'analogie (pp. 869-885). Paris: Hermès Lavoisier: Revue d'intelligence artificielle.

Duvignau, K. (2004). Architeture structurale et fonctionnelle du lexique verbal: la flexibilité sémantique comme principe fondamental de la cognition humaine et artificielle. Projeto não publicado, Universidade de Toulouse, França.

Fenson, L., Dale, P. S., Reznick, J. S., Bates, E., Thal, D. J., \& Pethick, S. J. (1994). Variability in early communicative development. Monographs of the Society for Research in Child Development, 59, 1-173.

Fernald, A., \& Morikawa, H. (1993). Common themes and cultural variations in Japanese and American mothers' speech to infants. Child Development, 64, 637-656.

Figueira, R. A. (1998). Os lineamentos das conjugações verbais na fala da criança. Multidirecionalidade do erro e heterogeneidade linguística. Letras de Hoje, 33, 73-80.

Figueira, R. A. (1999). Aquisição dos verbos prefixados por des em português. Palavra, 5, 190-211.

Gentner, D. (1978). On relational meaning: The acquisition of verb meaning. Child development, 49, 988-998.

Gentner, D., \& Boroditsky, L. (2001). Individualtion, relativity and early word learning. In M. Bowerman \& S. C. Levinson (Eds.), Language, culture, and cognition: Language acquisition and conceptual development (pp. 215-256). New York: Cambridge University Press.

Gentner, D., \& Rattermann M. J. (1991). Language and the career of similarity. In S. A. Gelman \& J. P. Byrnes (Eds.), Perspectives on language and thought: Interrelations in development (pp. 225-277). Cambridge, MA: Cambridge University Press.

Gillette, J., Gleitman, H., Gleitman, L., \& Lederer, A. (1999). Human simulations of vocabulary learning. Cognition, 73, $135-176$.

Glock, H. J. (2003). Quine and Davidson on language, thougt and reality. New York: Cambridge University Press.

Golinkoff, R. M., Hirsh-Pasek, K., Mervis, C. B., Frawley, W. B., \& Parillo, M. (1995). Lexical principles can be extended to the acquisition of verbs. In W. E. Merriman \& M. Tomasello (Eds.), Beyond names for things: Young children's acquisition of verbs (pp. 185-222). Hillsdale: Lawrence Erlbaum Associates. 
Golinkoff, R. M., Mervis, C. B., \& Hirsh-Pasek, K. (1994). Early object labels: The case for a developmental lexical principles framework. Journal of Child Language, 21, 125-155.

Gopnik, A., Choi, S., \& Baumberger, T. (1996). Cross-linguistic differences in early semantic and cognitive development. Cognitive Development, 11, 197-227.

Hollich, G. J., Hirsh-Pasek, K., Golinkoff, R., Brand, R., Brown, E., Chung, H.L., Hennon, E., \& Rocroi, C. (2000). Breaking the language barrier: An emergentist coalition model for the origins of word learning. Monographs of the Society for Research in Child Development, 65, 1-123.

Keren-Portnoy, T. (2006). Facilitation and practice in verb acquisition. Journal of child language, 36, 487-518.

Kidd, E., Lieven, E., \& Tomasello, M. (2006). Examining the role of lexical frequency in the processing of sentential complements. Cognitive Development, 21, 93-107.

Laville, C., \& Dione, J. (1999). A construção do saber. Porto Alegre: Artes Médicas.

Ma, W., McDonough, C., Lannon, R., Golinkoff, R. M., HirshPasek, K., \& Tardif, T. (2006). A mental image is worth a thousand verbs: Imageability predicts verb learning. Jean Piaget Society, Baltimore, MD.

Maguire, M., Hirsh-Pasek, K., \& Golinkoff, R. (2006). A unified theory of word learning: Putting verb acquisition in context. In K. Hirsh-Pasek \& R. Golinkoff (Eds.), Action meets word: How children learn verbs (pp. 364-392). New York: Oxford University Press.

Medin, D. L., \& Schaffer, M. M. (1978). Context theory of classification learning. Psychological Review, 85, 207-238.

Naschmias, C., \& Nascmias, D. (1996). Research methods in the social sciences. London: Arnold.

Pasquali, L. (2001). Manual de técnicas de exame psicológicoTEP: Fundamentos de Técnicas Psicológicas. São Paulo: Casa do Psicólogo/Conselho Federal de Psicologia.

Paivio, A., Yuille, J. C., \& Madigan, S. A. (1968). Concreteness, imagery and meaningfulness values for 925 nouns. Journal of Experimental Psychology, 76, 1-25.

Ping, C., Tonietto, L., Parente, M. A., Duvignau, K., \& Gaume, B. (2009). Does the relative verb advantage in Chinese children's productive vocabularies guarantee the conventional meaning of verbs? A cross-cultural study of action naming by Chinese and Brazilian children. Manuscrito não publicado.

Poulin-Dubois, D., \& Forbes, J. N. (2002). Toddlers' attention to intentions-in-action in learning novel action words. Developmental Psychology, 38, 104-114.

Rosch, E. (1975). Cognitive representations of semantic categories. Journal of Experimental Psychology: General, 104, 192-233.
Rosch, E., \& Lloyd, B. B. (1978). Cognition and categorization. New Jersey: Lawrence Erlbaum Associates.

Tardif, T. (1996). Nouns are not always learned before verbs: Evidence from Mandarin speaker's early vocabulary. Development Psychology, 32, 492-504.

Tardif, T. (2006). But are they really verbs? Chinese words for action. In K. Hirsh-Pasek \& R. Golinkoff (Eds.), Action meets word: How children learn verbs (pp. 477-498). New York: Oxford University Press.

Tardif, T., Gelman, S., \& Xu, F. (1999). Putting the "noun bias" in context: A comparison of English and Mandarin. Child Development, 70, 620-635.

Tomasello, M. (2003). Constructing a language: A usage-based theory of language acquisition. Cambridge: Harvard University Press.

Tonietto, L. (2005). Metáfora e analogia no processo de formação de conceitos: Um estudo sobre aproximações semânticas verbais em crianças pré-escolares. Dissertação de Mestrado, Universidade Federal do Rio Grande do Sul, Porto Alegre.

Tonietto, L., Parente, M. A. M. P., Duvignau, K., Gaume, B., \& Bosa, C. A. (2007). Aquisição inicial do léxico verbal e aproximações semânticas em português. Psicologia: Reflexão e Crítica, 20, 114-123.

Vidor, D. C. G. M. (2008). Aquisição lexical inicial por crianças falantes do Português Brasileiro: Discussão do fenômeno da explosão do vocabulário e da atuação da hipótese do viés nominal. Tese de Doutorado, Pontifícia Universidade Católica do Rio Grande do Sul, Porto Alegre.

Vygotsky, L. S. (1998). Pensamento e linguagem (J. L. Camargo, Trad.). São Paulo: Martins Fontes (Trabalho original publicado em 1987).

Wagner, L., Swensenb, L. D., \& Naigles, L. R. (2009). Children's early productivity in verbal morphology. Cognitive Development, 24, 223-239.

Recebido em 16.09.2009

Primeira decisão editorial em 18.08.2010

Versão final em 13.09.2010

Aceito em 13.09.2011 